\title{
SINGULAR PERTURBATIONS OF SOME NONLINEAR PROBLEMS
}

\author{
M. Chipot* \\ Institute of Mathematics, University of Zurich \\ Winterthurerstrasse 190, CH-8057, Zurich, Switzerland \\ m.m.chipot@math.uzh.ch

\section{S. Guesmia} \\ Institute of Mathematics, University of Zurich \\ Winterthurerstrasse 190, CH-8057, Zurich, Switzerland \\ Mathematics Section, ICTP \\ Strada Costiera 11, 34151 Trieste, Italy \\ senoussi.guesmia@math.uzh.ch \\ A. Sengouga \\ Department of Mathematics, University of M'sila \\ PB :166, 28000 M'sila, Algeria \\ amsengouga@yahoo.fr
}

UDC 519.6

Dedicated to Professor V. V. Zhikov on the occasion of his 70th birthday

We deal with singular perturbations of nonlinear problems depending on a small parameter $\varepsilon>0$. First we consider the abstract theory of singular perturbations of variational inequalities involving some nonlinear operators, defined in Banach spaces, and describe the asymptotic behavior of these solutions as $\varepsilon \rightarrow 0$. Then these abstract results are applied to some boundary value problems. Bibliography: 15 titles.

\section{Introduction}

The goal of this paper is to study the asymptotic behavior of singular perturbations problems as a parameter $\varepsilon$ goes towards 0 . Our results are very general, but we have more particularly in mind anisotropic cases where $\varepsilon$ only acts on some variables of a domain $\Omega \subset \mathbb{R}^{n}$ ( $n$ is an integer) where we consider the partial differential equations. To be more precise we can take, as a model, the diffusion problem defined in the unit square $\Omega=(0,1) \times(0,1)$

$$
\begin{cases}-\varepsilon^{2} \partial_{x_{1}}^{2} u_{\varepsilon}-\partial_{x_{2}}^{2} u_{\varepsilon}=f & \text { in } \Omega, \\ u_{\varepsilon}=0 & \text { on } \partial \Omega,\end{cases}
$$

\footnotetext{
* To whom the correspondence should be addressed.
}

Translated from Problems in Mathematical Analysis 58, June 2011, pp. 107-120.

1072-3374/11/1766-0828 (C) 2011 Springer Science+Business Media, Inc. 
where $\varepsilon>0$ and $f$ represents the source term. We assume that the diffusion in the $x_{1}$-direction is negligible with respect to the other direction as $\varepsilon \rightarrow 0$. Formally, the natural limit of $u_{\varepsilon}$ is a function $u_{0}$ defined on the sections $\left\{x_{1}\right\} \times(0,1)$ for a.e. $x_{1} \in(0,1)$ as a solution to the problem

$$
\left\{\begin{array}{l}
-\partial_{x_{2}}^{2} u_{0}\left(x_{1}, \cdot\right)=f\left(x_{1}, \cdot\right) \quad \text { in }(0,1), \\
u_{0}\left(x_{1}, \cdot\right)=0 \text { on }\{0,1\} .
\end{array}\right.
$$

Note that the variable $x_{1}$ plays a role of a parameter. It is clear that if $f$ (not identically equal to 0$)$ is independent of $x_{1}$, i.e., $f=f\left(x_{2}\right)$, then $u_{0} \notin H_{0}^{1}(\Omega)$. This prevents the convergence $u_{\varepsilon} \rightarrow u_{0}$ to occur in $H^{1}(\Omega)$. From this remark we may discuss many issues concerning this convergence.

In this paper, we begin by dealing with abstract singular perturbations problems of variational inequalities. Our approach has the advantage to include in a short theory a wide class of problems spread in the literature. We give then some applications of it.

In the literature, linear elliptic, parabolic, and hyperbolic problems defined on arbitrary domains are analyzed in different contexts and the convergence $u_{\varepsilon} \rightarrow u_{0}$ is obtained in different norms. A boundary layer may occur at the lateral boundary of cylindrical domains $(\{0,1\} \times(0,1)$ for the above example). The convergence in Sobolev spaces may be shown in regions far from this lateral boundary. We may see this clearly when our perturbed problem satisfies some cylindrical symmetries. This means that $f=f\left(x_{2}\right)$ in the above example. In this case, $u_{\varepsilon}$ converges towards $u_{0}$ at an exponential rate. For more details we refer the reader to [1]-[10].

An abstract approach to this theory was also given in $[11,12]$ where the following operator equation is considered:

$$
\varepsilon A u_{\varepsilon}+B u_{\varepsilon}=f
$$

with $A$ and $B$ linear operators defined on Hilbert spaces. This approach covers diagonal structure problems as the problem (1.1). The authors also showed, as in the case of partial differential equations, that $u_{\varepsilon}$ converges towards $u_{0}$ solution to the equation

$$
B u_{0}=f
$$

as $\varepsilon \rightarrow 0$. There are also some previous works on singular perturbations of variational inequalities, i.e., when (1.3) is replaced by

$$
\left(\varepsilon A u_{\varepsilon}, v-u_{\varepsilon}\right)+\left(B u_{\varepsilon}, v-u_{\varepsilon}\right) \geqslant\left(f, v-u_{\varepsilon}\right) \quad \forall v \in K
$$

where $K$ is some nonempty closed convex set (cf. [13]-[15]). In [15], this abstract approach is established to investigate the isotropic singular perturbations problems.

In order to cover a larger class of problems by an abstract theory, we deal with the variational inequality (1.4) when $A$ and $B$ are nonlinear operators defined on different Banach spaces $V$ and $W$ respectively, which, in particular, applies to the anisotropic singular perturbations problems. This is what we will see in the next section. In the last section, the first example is devoted to show that these results also cover the isotropic case. Then some examples of anisotropic singular perturbations problems are introduced in order to illustrate some points of the theory as, for instance, the lack of compactness.

\section{Abstract Singular Perturbations Problems}

Let $V$ and $W$ be two reflexive separable Banach spaces equipped with the norms $|\cdot|_{V}$ and | $\left.\cdot\right|_{W}$ respectively. We suppose that the space $V \cap W$ is dense in $V$ and $W$, and is equipped 
with the norm

$$
|\cdot|_{V \cap W}=|\cdot|_{V}+|\cdot|_{W} \cdot
$$

Of course, $V \cap W$ is a Banach space equipped with the previous norm. For any space $X$ we denote by $\langle\cdot, \cdot\rangle_{X}$ the duality pairing between $X^{\prime}$ and $X$, where $X^{\prime}$ is the dual of $X$. It is clear that

$$
V \cap W \subset V, W \quad \text { and } \quad V^{\prime}, W^{\prime} \subset(V \cap W)^{\prime} .
$$

Moreover, one can check that $(V \cap W)^{\prime}=V^{\prime}+W^{\prime}$. We consider two nonlinear operators $A$ and $B$ such that

$$
A: V \rightarrow V^{\prime}, \quad B: W \rightarrow W^{\prime} .
$$

We suppose that $A, B$ are monotone, that is to say that

$$
\begin{aligned}
& \langle A u-A v, u-v\rangle_{V} \geqslant 0 \quad \forall u, v \in V, \\
& \langle B u-B v, u-v\rangle_{W} \geqslant 0 \quad \forall u, v \in W .
\end{aligned}
$$

We denote by $K \neq \varnothing$ a closed convex set of $V \cap W$ and for $A, B$ we make the following coerciveness assumption. We suppose that for some $v_{0} \in K$

$$
\begin{aligned}
& \frac{\left\langle A u-A v_{0}, u-v_{0}\right\rangle_{V}}{\left|u-v_{0}\right|_{V}} \rightarrow+\infty \quad \text { as }\left|u-v_{0}\right|_{V} \rightarrow+\infty, \quad u \in K, \\
& \frac{\left\langle B u-B v_{0}, u-v_{0}\right\rangle_{W}}{\left|u-v_{0}\right|_{W}} \rightarrow+\infty \quad \text { as }\left|u-v_{0}\right|_{W} \rightarrow+\infty, \quad u \in K .
\end{aligned}
$$

Remark 2.1. If $K$ is bounded in $V$ (respectively, in $W$ ) we will not need the assumption (2.3) (respectively, (2.4)). Note also that for some $v_{0} \in K$ they are equivalent with

$$
\begin{aligned}
& \frac{\left\langle A u, u-v_{0}\right\rangle_{V}}{\left|u-v_{0}\right|_{V}} \rightarrow+\infty \quad \text { as }\left|u-v_{0}\right|_{V} \rightarrow+\infty, \quad u \in K, \\
& \frac{\left\langle B u, u-v_{0}\right\rangle_{W}}{\left|u-v_{0}\right|_{W}} \rightarrow+\infty \quad \text { as }\left|u-v_{0}\right|_{W} \rightarrow+\infty, \quad u \in K .
\end{aligned}
$$

In addition, we assume that

$$
\begin{aligned}
& A \text { sends bounded sets of } V \text { in bounded sets of } V^{\prime}, \\
& B \text { sends bounded sets of } W \text { in bounded sets of } W^{\prime}, \\
& A \text { and } B \text { are hemicontinuous on } V \text { and } W \text { respectively. }
\end{aligned}
$$

This last assumption means that - for instance, for $A-$

$$
t \mapsto\langle A(u+t v), w\rangle_{V} \text { is continuous on } \mathbb{R} \text { for all } u, v, w \in V \text {. }
$$

Under the assumptions above, we have the following assertion.

Theorem 2.1. For $f \in(V \cap W)^{\prime}$ and $\varepsilon>0$ there exists a solution $u_{\varepsilon}$ to the problem

$$
\left\{\begin{array}{l}
\varepsilon\left\langle A u_{\varepsilon}, v-u_{\varepsilon}\right\rangle_{V}+\left\langle B u_{\varepsilon}, v-u_{\varepsilon}\right\rangle_{W} \geqslant\left\langle f, v-u_{\varepsilon}\right\rangle_{V \cap W} \quad \forall v \in K, \\
u_{\varepsilon} \in K .
\end{array}\right.
$$

Moreover, if $A$ or $B$ is strictly monotone (i.e., if one of the inequalities (2.1), (2.2) is strict for $u \neq v)$, the solution is unique. 
Proof. We consider the operator $A_{\varepsilon}$ defined by

$$
A_{\varepsilon}: V \cap W \rightarrow(V \cap W)^{\prime}=V^{\prime}+W^{\prime}, \quad v \mapsto \varepsilon A v+B v .
$$

This operator is monotone, hemicontinuous, and coercive on $K$. For this last point, by the coerciveness assumptions on $A$ and $B$, for every $M>0$ there exist $\delta_{1}(M), \delta_{2}(M) \geqslant 1$ such that

$$
\begin{aligned}
& \left|u-v_{0}\right|_{V} \geqslant \delta_{1}(M) \Rightarrow \frac{\left\langle\varepsilon A u, u-v_{0}\right\rangle_{V}}{\left|u-v_{0}\right|_{V}} \geqslant M, \\
& \left|u-v_{0}\right|_{W} \geqslant \delta_{2}(M) \Rightarrow \frac{\left\langle B u, u-v_{0}\right\rangle_{W}}{\left|u-v_{0}\right|_{W}} \geqslant M .
\end{aligned}
$$

Since $A$ and $B$ are bounded, there exist constants $C_{A}$ and $C_{B}$ such that

$$
\begin{aligned}
& \left|u-v_{0}\right|_{V} \leqslant \delta_{1}(M) \Rightarrow\left|\left\langle\varepsilon A u, u-v_{0}\right\rangle_{V}\right| \leqslant C_{A}(M), \\
& \left|u-v_{0}\right|_{W} \leqslant \delta_{2}(M) \Rightarrow\left|\left\langle B u, u-v_{0}\right\rangle_{W}\right| \leqslant C_{B}(M) .
\end{aligned}
$$

Choose

$$
\left|u-v_{0}\right|_{V}+\left|u-v_{0}\right|_{W} \geqslant 2 \delta_{1}(M)+2 \delta_{2}(M)+\delta_{1}\left(2 M+2 C_{B}(M)\right)+\delta_{2}\left(2 M+2 C_{A}(M)\right) .
$$

Of course, one has either $\left|u-v_{0}\right|_{V} \geqslant \delta_{1}(M)$ or $\left|u-v_{0}\right|_{W} \geqslant \delta_{2}(M)$. Suppose, for instance, that $\left|u-v_{0}\right|_{V} \geqslant \delta_{1}(M)$, the other case being the same. If, moreover, $\left|u-v_{0}\right|_{W} \geqslant \delta_{2}(M)$, from (2.11) and (2.12) one has

$$
\begin{aligned}
\frac{\left\langle\varepsilon A u, u-v_{0}\right\rangle_{V}+\left\langle B u, u-v_{0}\right\rangle_{W}}{\left|u-v_{0}\right|_{V}+\left|u-v_{0}\right|_{W}} & =\frac{\left|u-v_{0}\right|_{V}}{\left|u-v_{0}\right|_{V}+\left|u-v_{0}\right|_{W}} \cdot \frac{\left\langle\varepsilon A u, u-v_{0}\right\rangle_{V}}{\left|u-v_{0}\right|_{V}} \\
& +\frac{\left|u-v_{0}\right|_{W}}{\left|u-v_{0}\right|_{V}+\left|u-v_{0}\right|_{W}} \cdot \frac{\left\langle B u, u-v_{0}\right\rangle_{W}}{\left|u-v_{0}\right|_{W}} \geqslant M .
\end{aligned}
$$

If $\left|u-v_{0}\right|_{W} \leqslant \delta_{2}(M)$, then $\left|u-v_{0}\right|_{V} \geqslant \delta_{2}(M), \delta_{1}\left(2 M+2 C_{B}(M)\right)$, so that

$$
\begin{aligned}
\frac{\left\langle\varepsilon A u, u-v_{0}\right\rangle_{V}+\left\langle B u, u-v_{0}\right\rangle_{W}}{\left|u-v_{0}\right|_{V}+\left|u-v_{0}\right|_{W}} & \geqslant \frac{\left|u-v_{0}\right|_{V}}{\left|u-v_{0}\right|_{V}+\left|u-v_{0}\right|_{W}}\left\{2 M+2 C_{B}(M)\right\}-C_{B}(M) \\
& \geqslant \frac{1}{2}\left\{2 M+2 C_{B}(M)\right\}-C_{B}(M) \geqslant M .
\end{aligned}
$$

This shows the coerciveness of $A_{\varepsilon}$. The existence of $u_{\varepsilon}$ follows from the classical theory of variational inequalities.

Remark 2.2. Let $K=V \cap W$. Taking $v=u_{\varepsilon} \pm w, w \in K$, one sees that $u_{\varepsilon}$ is a solution to the problem

$$
\left\{\begin{array}{l}
\varepsilon A u_{\varepsilon}+B u_{\varepsilon}=f, \\
u_{\varepsilon} \in V \cap W .
\end{array}\right.
$$

We are now interested in studying the behavior of $u_{\varepsilon}$ as $\varepsilon \rightarrow 0$. Note that this is not possible in general. Indeed, taking, for instance, $V$ a Hilbert space, $A=$ the identity, $B=0, f \in V^{\prime}=V$, we can see that the solution to $(2.13)$ is given by $u_{\varepsilon}=f / \varepsilon$ and $\left(u_{\varepsilon}\right)_{\varepsilon}$ has no limit. In what follows, we will assume that

$$
f \in W^{\prime} .
$$

The essential convergences are given as follows. 
Theorem 2.2. Suppose that $f \in W^{\prime}$ and $u_{\varepsilon}$ is a solution to (2.10). Then, as $\varepsilon \rightarrow 0$,

(i) $u_{\varepsilon}$ is bounded in $W$ independently of $\varepsilon$,

(ii) $\varepsilon u_{\varepsilon} \rightarrow 0$ in $V$,

(iii) $\varepsilon A u_{\varepsilon} \rightarrow 0$ in $V^{\prime}$,

(iv) $\left\langle\varepsilon A u_{\varepsilon}, u_{\varepsilon}\right\rangle_{V} \rightarrow 0$.

Proof. (i) Choose $v_{0} \in K$ such that (2.5) and (2.6) hold. Suppose that $\left|u_{\varepsilon}-v_{0}\right|_{W}$ is unbounded. Then for some sequence $\varepsilon_{k} \rightarrow 0$

$$
\left|u_{\varepsilon_{k}}-v_{0}\right|_{W} \rightarrow+\infty
$$

Taking $v=v_{0}$ in $(2.10)$, we derive

$$
\varepsilon_{k}\left\langle A u_{\varepsilon_{k}}, u_{\varepsilon_{k}}-v_{0}\right\rangle_{V}+\left\langle B u_{\varepsilon_{k}}, u_{\varepsilon_{k}}-v_{0}\right\rangle_{W} \leqslant\left\langle f, u_{\varepsilon_{k}}-v_{0}\right\rangle_{W} \leqslant|f|_{W^{\prime}}\left|u_{\varepsilon_{k}}-v_{0}\right|_{W} .
$$

It follows that

$$
\frac{\varepsilon_{k}\left\langle A u_{\varepsilon_{k}}, u_{\varepsilon_{k}}-v_{0}\right\rangle_{V}}{\left|u_{\varepsilon_{k}}-v_{0}\right|_{W}}+\frac{\left\langle B u_{\varepsilon_{k}}, u_{\varepsilon_{k}}-v_{0}\right\rangle_{W}}{\left|u_{\varepsilon_{k}}-v_{0}\right|_{W}} \leqslant|f|_{W^{\prime}} .
$$

If $\left|u_{\varepsilon_{k}}-v_{0}\right|_{V}$ is bounded, then

$$
\frac{\varepsilon_{k}\left\langle A u_{\varepsilon_{k}}, u_{\varepsilon_{k}}-v_{0}\right\rangle_{V}}{\left|u_{\varepsilon_{k}}-v_{0}\right|_{W}} \rightarrow 0
$$

else, by the coerciveness of $A$, this term is nonnegative for some $k$ large enough. In both cases, due to the coerciveness of $B$, the left-hand side of (2.15) is unbounded, which is impossible. This proves assertion (i).

(ii) Since $u_{\varepsilon}$ is bounded in $W$ and, consequently, $B u_{\varepsilon}$ is bounded in $W^{\prime}$, from (2.10) written for $v=v_{0}$ we derive that

$$
\varepsilon\left\langle A u_{\varepsilon}, u_{\varepsilon}-v_{0}\right\rangle_{V} \leqslant C
$$

for some constant $C$ independent of $\varepsilon$. If $\left(u_{\varepsilon}-v_{0}\right)$ is bounded in $V$, it is clear that $\varepsilon u_{\varepsilon}=$ $\varepsilon\left(u_{\varepsilon}-v_{0}\right)+\varepsilon v_{0} \rightarrow 0$. Also, from (2.5) and (2.16) we have - up to a subsequence -

$$
\varepsilon\left|u_{\varepsilon}-v_{0}\right|_{V} \leqslant C \frac{\left|u_{\varepsilon}-v_{0}\right|_{V}}{\left\langle A u_{\varepsilon}, u_{\varepsilon}-v_{0}\right\rangle_{V}} \rightarrow 0
$$

and the result follows as in the previous case.

(iii) and (iv) We first show that $\varepsilon A u_{\varepsilon} \rightarrow 0$ in $V^{\prime}$. Let $v \in V$. By the monotonicity of $A$,

$$
\varepsilon\left\langle A u_{\varepsilon}-A v, u_{\varepsilon}-v\right\rangle_{V} \geqslant 0
$$

Hence

$$
\varepsilon\left\langle A u_{\varepsilon}, v\right\rangle_{V} \leqslant \varepsilon\left\langle A u_{\varepsilon}, u_{\varepsilon}\right\rangle_{V}+\left\langle A v, \varepsilon\left(v-u_{\varepsilon}\right)\right\rangle_{V} .
$$

For $v_{0} \in K$ from (2.16) we derive

$$
\varepsilon\left\langle A u_{\varepsilon}, u_{\varepsilon}\right\rangle_{V} \leqslant\left\langle\varepsilon A u_{\varepsilon}, v_{0}\right\rangle_{V}+C .
$$

Using (2.18), we get

$$
\varepsilon\left\langle A u_{\varepsilon}, v-v_{0}\right\rangle_{V} \leqslant C+\left\langle A v, \varepsilon\left(v-u_{\varepsilon}\right)\right\rangle_{V},
$$


where $C$ is a constant independent of $\varepsilon$. Choosing $v \in v_{0}+\mathscr{B}_{1}$, where $\mathscr{B}_{1}$ is the unit ball of $V$, we arrive to

$$
\varepsilon\left\langle A u_{\varepsilon}, v_{1}\right\rangle_{V} \leqslant C^{\prime} \quad \forall v_{1} \in \mathscr{B}_{1},
$$

where $C^{\prime}$ is independent of $\varepsilon$. Thus, $\varepsilon A_{\varepsilon}$ is bounded in $V^{\prime}$ and - for some subsequence -

$$
\varepsilon A u_{\varepsilon} \rightarrow \psi \text { in } V^{\prime}
$$

Passing to the limit in (2.19) we derive

$$
\left\langle\psi, v-v_{0}\right\rangle_{V} \leqslant C \quad \forall v \in V
$$

Thus, $\psi=0$. By the uniqueness of the possible limits, we have shown that

$$
\varepsilon A u_{\varepsilon} \rightarrow 0 \text { in } V^{\prime}
$$

For any $v \in K$, by (2.10) and the monotonicity of $B$, we have

$$
\begin{aligned}
\varepsilon\left\langle A u_{\varepsilon}, u_{\varepsilon}\right\rangle_{V} & \leqslant\left\langle\varepsilon A u_{\varepsilon}, v\right\rangle_{V}+\left\langle f, u_{\varepsilon}-v\right\rangle_{W}+\left\langle B u_{\varepsilon}, v-u_{\varepsilon}\right\rangle_{W} \\
& \leqslant\left\langle\varepsilon A u_{\varepsilon}, v\right\rangle_{V}+\left\langle f, u_{\varepsilon}-v\right\rangle_{W}+\left\langle B v, v-u_{\varepsilon}\right\rangle_{W} .
\end{aligned}
$$

Let $\left(\varepsilon_{k}\right)_{k}$ be a sequence such that

$$
\varepsilon_{k}\left\langle A u_{\varepsilon_{k}}, u_{\varepsilon_{k}}\right\rangle_{V} \rightarrow \lim _{\varepsilon \rightarrow 0} \sup \varepsilon\left\langle A u_{\varepsilon}, u_{\varepsilon}\right\rangle_{V}
$$

Since $u_{\varepsilon_{k}}$ is bounded in $W$, one can suppose - extracting, if necessary, another subsequence that

$$
u_{\varepsilon_{k}} \rightarrow \widetilde{u} \quad \text { in } W
$$

Passing to the limit in $(2.20)$ written for $\varepsilon_{k}$, we get

$$
\lim _{\varepsilon \rightarrow 0} \sup \varepsilon\left\langle A u_{\varepsilon}, u_{\varepsilon}\right\rangle_{V} \leqslant\langle f, \widetilde{u}-v\rangle_{W}+\langle B v, v-\widetilde{u}\rangle_{W} \quad \forall v \in K
$$

It is clear that $\widetilde{u}$ belongs to $\bar{K}^{W}$, the weak closure of $K$ in $W$ which coincides with its strong closure since $K$ is convex. Thus, there exists a sequence $v_{n} \in K$ such that

$$
v_{n} \rightarrow \widetilde{u} \quad \text { in } W
$$

Taking $v=v_{n}$ in (2.21) and passing to the limit, we derive

$$
\lim _{\varepsilon \rightarrow 0} \sup \varepsilon\left\langle A u_{\varepsilon}, u_{\varepsilon}\right\rangle_{V} \leqslant 0 .
$$

Passing to the limit in (2.18), we also have

$$
\lim _{\varepsilon \rightarrow 0} \inf \varepsilon\left\langle A u_{\varepsilon}, u_{\varepsilon}\right\rangle_{V} \geqslant 0
$$

which proves (iv).

To complete the proof, going back to (2.18), for every $v_{1} \in \mathscr{B}_{1}$ one has

$$
\varepsilon\left\langle A u_{\varepsilon}, v_{1}\right\rangle_{V} \leqslant \varepsilon\left\langle A u_{\varepsilon}, u_{\varepsilon}\right\rangle_{V}+\left|A v_{1}\right|_{V^{\prime}}\left(\varepsilon+\left|\varepsilon u_{\varepsilon}\right|_{V}\right) \leqslant \varepsilon\left\langle A u_{\varepsilon}, u_{\varepsilon}\right\rangle_{V}+C\left(\varepsilon+\left|\varepsilon u_{\varepsilon}\right|_{V}\right) \rightarrow 0
$$

where $C$ is independent of $v_{1}$. This completes the proof of the theorem. 
Remark 2.3. In the case where $K=V \cap W$, from Equation (2.13) one derives that

$$
B u_{\varepsilon}-f \rightarrow 0 \text { in } V^{\prime} .
$$

In addition, we have the following assertion.

Theorem 2.3. Suppose that for some sequence $\varepsilon_{k} \rightarrow 0$

$$
u_{\varepsilon_{k}} \rightarrow \widetilde{u} \quad \text { in } W .
$$

Then $\widetilde{u}$ is a solution to the variational inequality

$$
\left\{\begin{array}{l}
\langle B \widetilde{u}, v-\widetilde{u}\rangle_{W} \geqslant\langle f, v-\widetilde{u}\rangle_{W} \quad \forall v \in \bar{K}^{W}, \\
\widetilde{u} \in \bar{K}^{W} .
\end{array}\right.
$$

Moreover,

$$
B u_{\varepsilon_{k}} \rightarrow B \widetilde{u} \quad \text { in } W^{\prime}, \quad\left\langle B u_{\varepsilon_{k}}, u_{\varepsilon_{k}}\right\rangle_{W} \rightarrow\langle B \widetilde{u}, \widetilde{u}\rangle_{W}
$$

Proof. Up to a subsequence - still labelled by $\varepsilon_{k}$ - one can assume that

$$
B u_{\varepsilon_{k}} \rightarrow \chi \quad \text { in } W^{\prime} \text {. }
$$

Passing to the limit in (2.10) written for $\varepsilon_{k}$, we obtain (cf. Theorem 2.2)

$$
\lim _{\varepsilon_{k} \rightarrow 0} \sup \left\langle B u_{\varepsilon_{k}}, u_{\varepsilon_{k}}\right\rangle_{W} \leqslant\langle\chi, v\rangle_{W}+\langle f, \widetilde{u}-v\rangle_{W} \quad \forall v \in K .
$$

Considering a sequence $v=v_{n} \rightarrow \widetilde{u}$ as above, we obtain

$$
\lim _{\varepsilon_{k} \rightarrow 0} \sup \left\langle B u_{\varepsilon_{k}}, u_{\varepsilon_{k}}\right\rangle_{W} \leqslant\langle\chi, \widetilde{u}\rangle_{W} .
$$

From the monotonicity of $B$ we have

$$
\left\langle B u_{\varepsilon_{k}}, u_{\varepsilon_{k}}\right\rangle_{W} \geqslant\left\langle B u_{\varepsilon_{k}}, v\right\rangle_{W}+\left\langle B v, u_{\varepsilon_{k}}-v\right\rangle_{W} \quad \forall v \in W .
$$

Then

$$
\lim _{\varepsilon_{k} \rightarrow 0} \inf \left\langle B u_{\varepsilon_{k}}, u_{\varepsilon_{k}}\right\rangle_{W} \geqslant\langle\chi, v\rangle_{W}+\langle B v, \widetilde{u}-v\rangle_{W} \quad \forall v \in W .
$$

It follows - taking $v=\widetilde{u}$ - that

$$
\lim _{\varepsilon_{k} \rightarrow 0}\left\langle B u_{\varepsilon_{k}}, u_{\varepsilon_{k}}\right\rangle_{W}=\langle\chi, \widetilde{u}\rangle_{W}
$$

From (2.27) we derive

$$
\langle\chi-B v, \widetilde{u}-v\rangle_{W} \geqslant 0 \quad \forall v \in W .
$$

Replacing $v$ by $\widetilde{u}+t w$ and letting $t \rightarrow 0$, we obtain

$$
\langle\chi-B \widetilde{u}, w\rangle_{W} \geqslant 0 \quad \forall w \in W,
$$

i.e., $\chi=B \widetilde{u}$. It follows that the whole sequence $B u_{\varepsilon_{k}}$ converges toward $B \widetilde{u}$. Moreover, (2.26) becomes

$$
\langle B \widetilde{u}, v-\widetilde{u}\rangle_{W} \geqslant\langle f, v-\widetilde{u}\rangle_{W} \quad \forall v \in K .
$$

Since $\bar{K}^{W}$ is closed (weakly closed), $\widetilde{u} \in \bar{K}^{W}$ and the above inequality holds also for every $v \in \bar{K}^{W}$. This completes the proof of the theorem. 
Remark 2.4. (i) We have proved that the only possible limits for the subsequences of $\left(u_{\varepsilon}\right)_{\varepsilon}$ are solutions to the variational inequality (2.24). In particular, if the solution is unique, then

$$
\begin{aligned}
& u_{\varepsilon} \rightarrow \widetilde{u} \text { in } W, \\
& B u_{\varepsilon} \rightarrow B \widetilde{u} \text { in } W^{\prime} .
\end{aligned}
$$

This is the case where $B$ is strictly monotone.

(ii) In the case where $K=V \cap W$, we have $\bar{K}^{W}=W$ and $\widetilde{u}$ is a solution to the equation

$$
B \widetilde{u}=f .
$$

Corollary 2.1. (i) Suppose that $A$ is strongly coercive in the sense that

$$
\langle A v, v\rangle_{V} \geqslant \lambda|v|_{V}^{\alpha} \quad \forall v \in V
$$

for some constants $\lambda>0$ and $\alpha>1$. Then

$$
\varepsilon^{1 / \alpha} u_{\varepsilon} \rightarrow 0 \quad \text { in } V
$$

(ii) If $B$ is strongly monotone in the sense that for some $\delta>0$ and $\beta>1$

$$
\langle B u-B v, u-v\rangle_{W} \geqslant \delta|u-v|_{W}^{\beta} \quad \forall v, u \in W,
$$

then the solution $\widetilde{u}$ to $(2.24)$ is unique and

$$
u_{\varepsilon} \rightarrow \widetilde{u} \quad \text { in } W .
$$

Proof. (i) follows directly from the inequality

$$
\varepsilon\left\langle A u_{\varepsilon}, u_{\varepsilon}\right\rangle_{V} \geqslant \lambda \varepsilon\left|u_{\varepsilon}\right|_{V}^{\alpha}
$$

and Theorem 2.2, (iv).

For (ii), by (2.30), $u_{\varepsilon} \in K$, and (2.25), one has

$$
\begin{aligned}
\delta\left|\widetilde{u}-u_{\varepsilon}\right|_{W}^{\beta} & \leqslant\left\langle B \widetilde{u}-B u_{\varepsilon}, \widetilde{u}-u_{\varepsilon}\right\rangle_{W} \leqslant\left\langle f, \widetilde{u}-u_{\varepsilon}\right\rangle_{W}-\left\langle B u_{\varepsilon}, \widetilde{u}-u_{\varepsilon}\right\rangle_{W} \\
& =\left\langle f, \widetilde{u}-u_{\varepsilon}\right\rangle_{W}+\left\langle B u_{\varepsilon}, u_{\varepsilon}\right\rangle_{W}-\left\langle B u_{\varepsilon}, \widetilde{u}\right\rangle_{W} \rightarrow 0 .
\end{aligned}
$$

Remark 2.5. If only the basic coerciveness (2.3) of $A$ is assumed, then the convergence result (ii) is sharp since, if $\alpha$ approaches 1 in (2.29), the exponent of $\varepsilon$ tends to 1 .

In the following assertion, some monotonicity property of $\left(u_{\varepsilon}\right)_{\varepsilon}$ is shown.

Corollary 2.2. Let $\varepsilon>\varepsilon^{\prime}>0$. Then

$$
\left\langle A u_{\varepsilon}, u_{\varepsilon}\right\rangle_{V} \leqslant\left\langle A u_{\varepsilon}, u_{\varepsilon^{\prime}}\right\rangle_{V} .
$$

Proof. Indeed, setting $v=u_{\varepsilon}$ (respectively, $v=u_{\varepsilon^{\prime}}$ ) in (2.10), written for $\varepsilon$ (respectively, $\varepsilon^{\prime}$ ), we get

$$
\varepsilon\left\langle A u_{\varepsilon}, u_{\varepsilon}-u_{\varepsilon^{\prime}}\right\rangle_{V}-\varepsilon^{\prime}\left\langle A u_{\varepsilon^{\prime}}, u_{\varepsilon}-u_{\varepsilon^{\prime}}\right\rangle_{V}+\left\langle B u_{\varepsilon}-B u_{\varepsilon^{\prime}}, u_{\varepsilon}-u_{\varepsilon^{\prime}}\right\rangle_{W} \leqslant 0 .
$$

Using the monotonicity of $A$ and $B$, it comes

$$
\varepsilon\left\langle A u_{\varepsilon}, u_{\varepsilon}-u_{\varepsilon^{\prime}}\right\rangle_{V} \leqslant \varepsilon^{\prime}\left\langle A u_{\varepsilon}, u_{\varepsilon}-u_{\varepsilon^{\prime}}\right\rangle_{V}-\varepsilon^{\prime}\left\langle A u_{\varepsilon}-A u_{\varepsilon^{\prime}}, u_{\varepsilon}-u_{\varepsilon^{\prime}}\right\rangle_{V} \leqslant \varepsilon^{\prime}\left\langle A u_{\varepsilon}, u_{\varepsilon}-u_{\varepsilon^{\prime}}\right\rangle_{V} .
$$

Then (2.31) follows because $\varepsilon>\varepsilon^{\prime}$. 
Remark 2.6. The above characterization is more clear if $A$ is linear. For instance, if $V$ is a Hilbert space and $A=I_{d}$, then (2.31) yields

$$
\left|u_{\varepsilon}\right|_{V} \leqslant\left|u_{\varepsilon^{\prime}}\right|_{V} \quad \text { for } \varepsilon^{\prime}<\varepsilon
$$

Next we pay attention to more regular problems, i.e., when some solutions to (2.24) are in $V$.

Corollary 2.3. If the variational inequality (2.24) has a solution $\widehat{u} \in K$ satisfying

$$
\liminf \langle A u, u-\widehat{u}\rangle_{V}>0 \text { as }|u|_{V} \rightarrow+\infty, \quad u \in K,
$$

then $u_{\varepsilon}$ is bounded in $V$ and there exists a sequence $u_{\varepsilon_{k}}$ such that

$$
u_{\varepsilon_{k}} \rightarrow \widetilde{u} \text { in } V \text { and } W,
$$

where $\widetilde{u} \in K$ is a solution to (2.24), i.e., all the accumulation points of $\left(u_{\varepsilon}\right)_{\varepsilon}$ belong to $K$ and are solutions to $(2.24)$.

In addition, if $B$ satisfies (2.30), then

$$
\left|u_{\varepsilon}-\widetilde{u}\right|_{W}=o\left(\varepsilon^{1 / \beta}\right) .
$$

Proof. Taking $v=\widehat{u}$ in (2.10), we derive

$$
\varepsilon\left\langle A u_{\varepsilon}, u_{\varepsilon}-\widehat{u}\right\rangle_{V} \leqslant\left\langle f, u_{\varepsilon}-\widehat{u}\right\rangle_{W}-\left\langle B u_{\varepsilon}, u_{\varepsilon}-\widehat{u}\right\rangle_{W} \leqslant-\left\langle B u_{\varepsilon}-B \widehat{u}, u_{\varepsilon}-\widehat{u}\right\rangle_{W} \leqslant 0 .
$$

Thus, $\left\langle A u_{\varepsilon}, u_{\varepsilon}-\widehat{u}\right\rangle_{V} \leqslant 0$ for all $\varepsilon>0$ and

$$
\lim _{\varepsilon \rightarrow 0} \sup \left\langle A u_{\varepsilon}, u_{\varepsilon}-\widehat{u}\right\rangle_{V} \leqslant 0 .
$$

By $(2.32), u_{\varepsilon}$ must be bounded in $V$, and one can find a sequence $\varepsilon_{k}$ such that

$$
u_{\varepsilon_{k}} \rightarrow \widetilde{u} \text { in } W, V \text {, and } V \cap W .
$$

In fact, since $u_{\varepsilon_{k}}$ is bounded in $V, W$, and $W \cap V$ one can assume that - up to a subsequence -

$$
u_{\varepsilon_{k}} \rightarrow u \text { in } V, \quad u_{\varepsilon_{k}} \rightarrow u^{\prime} \text { in } W, \quad u_{\varepsilon_{k}} \rightarrow u^{\prime \prime} \text { in } V \cap W .
$$

If $h \in V^{\prime} \subset V^{\prime}+W^{\prime}$, then

$$
\left\langle h, u_{\varepsilon_{k}}\right\rangle_{V \cap W} \rightarrow\langle h, u\rangle_{V \cap W}, \quad\left\langle h, u_{\varepsilon_{k}}\right\rangle_{V \cap W} \rightarrow\left\langle h, u^{\prime \prime}\right\rangle_{V \cap W}
$$

Hence

$$
\langle h, u\rangle_{V}=\left\langle h, u^{\prime \prime}\right\rangle_{V} \quad \forall h \in V^{\prime}
$$

Similarly, one can show that

$$
\left\langle h, u^{\prime}\right\rangle_{W}=\left\langle h, u^{\prime \prime}\right\rangle_{W} \quad \forall h \in W^{\prime}
$$

It follows that

$$
u=u^{\prime}=u^{\prime \prime}=\widetilde{u}
$$

and $\widetilde{u}$ is necessarily a solution to $(2.24)$.

For the last part of the corollary, since $\widehat{u}=\widetilde{u}$, by the uniqueness of the solution to (2.24), from $(2.35)$, one has

$$
\begin{aligned}
\delta\left|\widetilde{u}-u_{\varepsilon}\right|_{W}^{\beta} & \leqslant\left\langle B \widetilde{u}-B u_{\varepsilon}, \widetilde{u}-u_{\varepsilon}\right\rangle_{W} \leqslant-\varepsilon\left\langle A u_{\varepsilon}, u_{\varepsilon}-\widetilde{u}\right\rangle_{V} \\
& =-\varepsilon\left\langle A u_{\varepsilon}-A \widetilde{u}, u_{\varepsilon}-\widetilde{u}\right\rangle_{V}+\varepsilon\left\langle A \widetilde{u}, u_{\varepsilon}-\widetilde{u}\right\rangle_{V} \leqslant \varepsilon\left\langle A \widetilde{u}, u_{\varepsilon}-\widetilde{u}\right\rangle_{V}=o(\varepsilon),
\end{aligned}
$$

and the result follows. 
Remark 2.7. If we assume that $f=0, B(0)=0,0 \in K$, and $B$ satisfies a hypothesis as (2.28), then $u_{\varepsilon} \rightarrow 0$ in $W$. Indeed, taking $v=0$ in (2.10)

$$
\varepsilon\left\langle A u_{\varepsilon}, u_{\varepsilon}\right\rangle_{V}+\left\langle B u_{\varepsilon}, u_{\varepsilon}\right\rangle_{W} \leqslant 0,
$$

and by the monotonicity of $A$ we have

$$
\lambda\left|u_{\varepsilon}\right|_{W}^{\beta} \leqslant \varepsilon\left\langle A u_{\varepsilon}-A(0), u_{\varepsilon}\right\rangle_{V}+\left\langle B u_{\varepsilon}, u_{\varepsilon}\right\rangle_{W} \leqslant-\varepsilon\left\langle A(0), u_{\varepsilon}\right\rangle_{V} .
$$

The convergence follows by Theorem 2.2.

\section{Some Applications}

It is interesting to note that, using a priori estimates in the previous section, there is no need to have some compactness assumptions to pass to the limit in the nonlinear terms. In order to illustrate this, we consider here three nonlinear elliptic boundary value problems as examples of the abstract theory above. We will apply the theory to some anisotropic singular perturbations problems in the last two examples. To also see the power of our abstract analysis in general, we consider a very classical case of nonlinear obstacle problems.

\subsection{Nonlinear obstacle problems}

We denote by $a(\xi)=\left(a_{i}(\xi)\right)$ a continuous vector field in $\mathbb{R}^{n}$. We suppose that $a$ is such that for some $\lambda, \Lambda>0$ and $c \in \mathbb{R}$

$$
\begin{aligned}
& a(\xi) \cdot \xi \geqslant \lambda|\xi|^{2}+c, \\
& |a(\xi)| \leqslant \Lambda|\xi| \quad \forall \xi \in \mathbb{R}^{n}
\end{aligned}
$$

and, in addition,

$$
(a(\xi)-a(\zeta)) \cdot(\xi-\zeta) \geqslant 0 \quad \forall \xi, \zeta \in \mathbb{R}^{n} .
$$

Then, for $f \in L^{2}(\Omega)$ there exists a unique solution $u_{\varepsilon}$ to

$$
\left\{\begin{array}{l}
u_{\varepsilon} \in K_{0}=\left\{v \in H_{0}^{1}(\Omega) \mid v(x) \geqslant 0, \quad \text { a.e. } x \in \Omega\right\}, \\
\varepsilon \int_{\Omega} a\left(\nabla u_{\varepsilon}\right) \cdot \nabla\left(v-u_{\varepsilon}\right) d x+\int_{\Omega} u_{\varepsilon}\left(v-u_{\varepsilon}\right) d x \geqslant \int_{\Omega} f\left(v-u_{\varepsilon}\right) d x \quad \forall v \in K_{0},
\end{array}\right.
$$

where $\Omega$ is a bounded open subset in $\mathbb{R}^{n}$. Then setting

$$
V=H_{0}^{1}(\Omega), \quad W=L^{2}(\Omega), \quad A u=-\operatorname{div}(a(\nabla u)), \quad B=I_{d},
$$

our results apply and we get

$$
u_{\varepsilon} \rightarrow f^{+} \quad \text { in } L^{2}(\Omega)
$$

where $f^{+}$(respectively, $f^{-}$) denotes the positive (respectively, negative) part of $f$. Indeed, thanks to Theorems 2.2, 2.3 and Corollary 2.1 we see that $u_{\varepsilon} \rightarrow \widetilde{u}$ in $L^{2}(\Omega)$ where $\widetilde{u}$ is the unique solution to the problem

$$
\left\{\begin{array}{l}
\widetilde{u} \in \bar{K}_{0}=\left\{v \in L^{2}(\Omega) \mid v(x) \geqslant 0, \quad \text { a.e. } x \in \Omega\right\} \\
\int_{\Omega} \widetilde{u}(v-\widetilde{u}) d x \geqslant \int_{\Omega} f(v-\widetilde{u}) d x \quad \forall v \in \bar{K}_{0}
\end{array}\right.
$$


But it is clear that

$$
\begin{aligned}
\int_{\Omega} f^{+}\left(v-f^{+}\right) d x & =\int_{\Omega}\left(f+f^{-}\right)\left(v-f^{+}\right) d x \\
& =\int_{\Omega} f\left(v-f^{+}\right) d x+\int_{\Omega} f^{-} v d x \geqslant \int_{\Omega} f\left(v-f^{+}\right) d x \quad \forall v \in \bar{K}_{0}
\end{aligned}
$$

and $\widetilde{u}=f^{+}$. As a consequence of Theorems 2.2, 2.3 and Corollary 2.1 we can state the following.

Corollary 3.1. As $\varepsilon \rightarrow 0$, we have

$$
\begin{aligned}
& u_{\varepsilon} \rightarrow f^{+} \quad \text { in } L^{2}(\Omega), \quad \varepsilon u_{\varepsilon} \rightarrow 0 \quad \text { in } H_{0}^{1}(\Omega), \\
& -\varepsilon \partial_{x_{i}}\left(a\left(\nabla u_{\varepsilon}\right)\right) \rightarrow 0 \quad \text { in } H^{-1}(\Omega), \quad i=1, \cdots, n, \\
& \varepsilon \int_{\Omega} a\left(\nabla u_{\varepsilon}\right) \cdot \nabla u_{\varepsilon} d x \rightarrow 0 .
\end{aligned}
$$

Remark 3.1. Note that, as in (3.1), we may add a constant $c \in \mathbb{R}$ in (2.28) since it will be neglected once it is multiplied by $\varepsilon$, i.e.,

$$
\langle A v, v\rangle_{V} \geqslant \lambda|v|_{V}^{\alpha}+c \quad \forall v \in V .
$$

Of course, here the strong convergence of $\sqrt{\varepsilon} \nabla u_{\varepsilon}$ comes from the last convergence in the above corollary, i.e., $\sqrt{\varepsilon} \nabla u_{\varepsilon} \rightarrow 0$ in $L^{2}(\Omega)$.

\subsection{Semilinear elliptic problems}

Let $\Omega$ be a bounded open subset of $\mathbb{R}^{n}$ with sufficiently smooth boundary. We split the components of a point $x \in \mathbb{R}^{n}$ into the $q$ first components and the $n-q$ last ones, i.e.,

$$
X_{1}=\left(x_{1}, \ldots, x_{q}\right) \quad \text { and } \quad X_{2}=\left(x_{q+1}, \ldots, x_{n}\right) \text {, }
$$

where $q$ is a positive integer such that $q<n$. We denote by $\Pi_{X_{1}}$ (respectively, $\Pi_{X_{2}}$ ) the orthogonal projection from $\mathbb{R}^{n}$ onto the space $X_{2}=0$ (respectively, $X_{1}=0$ ). For any $X_{1} \in$ $\Pi_{1}:=\Pi_{X_{1}}(\Omega)$ and $X_{2} \in \Pi_{2}:=\Pi_{X_{2}}(\Omega)$ we denote by $\Omega_{X_{1}}$ (respectively, $\Omega_{X_{2}}$ ) the section of $\Omega$ above $X_{1}$ (respectively, $X_{2}$ ) i.e.,

$$
\Omega_{X_{1}}=\left\{X_{2} \mid\left(X_{1}, X_{2}\right) \in \Omega\right\} \quad \text { and } \quad \Omega_{X_{2}}=\left\{X_{1} \mid\left(X_{1}, X_{2}\right) \in \Omega\right\} .
$$

With this notation we set

$$
\nabla u=\left(\partial_{x_{1}} u, \ldots, \partial_{x_{n}} u\right)^{T}=\left(\begin{array}{c}
\left(\partial_{x_{1}} u, \ldots, \partial_{x_{q}} u\right)^{T} \\
\left(\partial_{x_{q+1}} u, \ldots, \partial_{x_{n}} u\right)^{T}
\end{array}\right)=\left(\begin{array}{c}
\nabla_{X_{1}} u \\
\nabla_{X_{2}} u
\end{array}\right) .
$$

We consider the following semilinear elliptic problem:

$$
\left\{\begin{array}{l}
-\varepsilon \Delta_{X_{1}} u_{\varepsilon}-\Delta_{X_{2}} u_{\varepsilon}+g\left(x, u_{\varepsilon}\right)=f \quad \text { in } \Omega, \\
u_{\varepsilon} \in H_{0}^{1}(\Omega) \cap L^{p}(\Omega),
\end{array}\right.
$$


where

$$
\Delta_{X_{1}}=\sum_{i=1}^{i=q} \frac{\partial^{2}}{\partial^{2} x_{i}}, \quad \Delta_{X_{2}}=\sum_{i=q+1}^{i=n} \frac{\partial^{2}}{\partial^{2} x_{i}}, \quad p>1, \quad f \in L^{2}(\Omega)+L^{p^{\prime}}(\Omega),
$$

where $p^{\prime}$ is the conjugate of $p$. In order to apply the abstract approach, we assume that $g$ : $\Omega \times \mathbb{R} \rightarrow \mathbb{R}$ is a Carathédeory function and nondecreasing in the second variable, i.e.,

$$
\begin{aligned}
x & \mapsto g(x, t) \text { is measurable on } \Omega \text { for all } t \in \mathbb{R}, \\
t & \mapsto g(x, t) \text { is continuous and nondecreasing on } \mathbb{R} \text { for a.e. } x \in \Omega
\end{aligned}
$$

and there exist $c, c^{\prime} \geqslant 0$ such that

$$
\begin{aligned}
& |g(x, t)| \leqslant c|t|^{p-1}+c^{\prime} \quad \forall t \in \mathbb{R} \quad \text { a.e. } x \in \Omega, \\
& g(x, t) t \geqslant|t|^{p} \quad \forall t \in \mathbb{R} \quad \text { a.e. } x \in \Omega .
\end{aligned}
$$

It is clear that, if $u \in L^{p}(\Omega)$, then $g(\cdot, u(\cdot)) \in L^{p^{\prime}}(\Omega)$. So $g$ defines an operator (still labelled by $g$ ) from $L^{p}(\Omega)$ into $L^{p^{\prime}}(\Omega)$ by

$$
u \mapsto g(\cdot, u(\cdot)),
$$

which is bounded, monotone and hemicontinuous. Then we choose the suitable Banach spaces

$$
V=\left\{\begin{array}{l|l}
u \in L^{2}(\Omega) & \begin{array}{l}
\nabla_{X_{1}} u \in\left[L^{2}(\Omega)\right]^{q}, \\
u\left(\cdot, X_{2}\right) \in H_{0}^{1}\left(\Omega_{X_{2}}\right), \text { a.e. } X_{2} \in \Pi_{2}
\end{array}
\end{array}\right\},
$$

equipped with the norm

$$
|v|_{V}:=\left|\nabla_{X_{1}} v\right|_{L^{2}(\Omega)}
$$

and

$$
W=\left\{\begin{array}{l|l}
u \in L^{2}(\Omega) \cap L^{p}(\Omega) \mid \begin{array}{l}
\nabla_{X_{2}} u \in\left[L^{2}(\Omega)\right]^{n-q}, \\
u\left(X_{1}, \cdot\right) \in H_{0}^{1}\left(\Omega_{X_{1}}\right)
\end{array} \quad \text { a.e. } X_{1} \in \Pi_{1}
\end{array}\right\},
$$

equipped with the norm

$$
|v|_{W}:=\left|\nabla_{X_{2}} v\right|_{L^{2}(\Omega)}+|v|_{L^{p}(\Omega)} .
$$

We can easily check that $V$ and $W$ are separable reflexive Banach spaces. Next we set

$$
A=-\Delta_{X_{1}} \quad \text { and } \quad B=-\Delta_{X_{2}}+g(x, \cdot) .
$$

Then the operator $A: V \rightarrow V^{\prime}$ is linear, bounded, and coercive. Since the operator $B: W \rightarrow W^{\prime}$ is a sum of a linear operator, satisfying the same properties as $A$, and the operator defined in (3.8), it is bounded, monotone, and coercive. In this example, the limit problem is defined for a.e. $X_{1} \in \Pi_{1}$ as

$$
\left\{\begin{array}{l}
-\Delta_{X_{2}} \widetilde{u}\left(X_{1}, \cdot\right)+g\left(\left(X_{1}, \cdot\right), \widetilde{u}\left(X_{1}, \cdot\right)\right)=f\left(X_{1}, \cdot\right) \text { in } \Omega_{X_{1}}, \\
\widetilde{u}\left(X_{1}, \cdot\right)=0 \text { on } \partial \Omega_{X_{1}} .
\end{array}\right.
$$

Then it remains to precise the connection between the boundary conditions, which is the subject of the following proposition.

Proposition 3.1. Let $V$ and $W$ be the spaces defined in (3.9) and (3.10) respectively. Then, if the boundary of $\Omega$ is smooth, we have

$$
V \cap W=H_{0}^{1}(\Omega) \cap L^{p}(\Omega) .
$$


Proof. The first inclusion $H_{0}^{1}(\Omega) \cap L^{p}(\Omega) \subset V \cap W$ is easy. For $u \in H_{0}^{1}(\Omega) \cap L^{p}(\Omega)$ there exists a sequence $\left(u_{n}\right)_{n} \subset \mathscr{D}(\Omega)$ such that $u_{n} \rightarrow u$ in $H_{0}^{1}(\Omega) \cap L^{p}(\Omega)$. In particular, we have

$$
\left|\nabla\left(u_{n}-u\right)\right|_{L^{2}(\Omega)} \rightarrow 0 .
$$

By the Lebesgue theorem, we get - up to a subsequence - for a.e. $X_{1} \in \Pi_{1}$ and $X_{2} \in \Pi_{2}$

$$
\begin{aligned}
& \left|\nabla\left(u_{n}\left(X_{1}, \cdot\right)-u\left(X_{1}, \cdot\right)\right)\right|_{L^{2}\left(\Omega_{X_{1}}\right)} \rightarrow 0, \\
& \left|\nabla\left(u_{n}\left(\cdot, X_{2}\right)-u\left(\cdot, X_{2}\right)\right)\right|_{L^{2}\left(\Omega_{X_{2}}\right)} \rightarrow 0 .
\end{aligned}
$$

This means that $u \in V$ and $u \in W$.

For the converse inclusion, we take $u \in V \cap W$ and consider the elliptic problem

$$
\left\{\begin{array}{l}
-\varepsilon \Delta v_{\varepsilon}+v_{\varepsilon}=u \text { in } \Omega \\
v_{\varepsilon}=0 \quad \text { on } \partial \Omega
\end{array}\right.
$$

Since $\Omega$ is sufficiently regular and of course $V \cap W \subset H^{1}(\Omega) \cap L^{p}(\Omega)$, we have $v_{\varepsilon} \in H^{2}(\Omega)$. According to Corollary 2.1, we derive

$$
v_{\varepsilon} \rightarrow u \quad \text { in } L^{2}(\Omega) .
$$

Then, applying the Laplace operator to the first equation in (3.12) and taking $-v_{\varepsilon}$ as a test function, we obtain

$$
\varepsilon\left\langle\Delta^{2} v_{\varepsilon}, v_{\varepsilon}\right\rangle_{H_{0}^{1}(\Omega)}-\int_{\Omega} \Delta v_{\varepsilon} v_{\varepsilon} d x=-\left\langle\Delta u, v_{\varepsilon}\right\rangle_{H_{0}^{1}(\Omega)} .
$$

It is clear that $\Delta u \in H^{-1}(\Omega), \Delta^{2} v_{\varepsilon} \in H^{-1}(\Omega)$ since

$$
-\Delta v_{\varepsilon}=\frac{u-v_{\varepsilon}}{\varepsilon} \in H^{1}(\Omega) .
$$

It follows that

$$
-\varepsilon \int_{\Omega} \nabla\left(\Delta v_{\varepsilon}\right) \cdot \nabla v_{\varepsilon} d x+\left|\nabla v_{\varepsilon}\right|_{L^{2}(\Omega)}^{2}=\int_{\Omega} \nabla u \cdot \nabla v_{\varepsilon} d x
$$

Hence

$$
\begin{aligned}
& -\varepsilon \int_{\Pi_{1}} \int_{\Omega_{X_{2}}} \nabla_{X_{1}}\left(\Delta v_{\varepsilon}\right) \cdot \nabla_{X_{1}} v_{\varepsilon} d X_{1} d X_{2}-\varepsilon \int_{\Pi_{2}} \int_{\Omega_{X_{1}}} \nabla_{X_{2}}\left(\Delta v_{\varepsilon}\right) \cdot \nabla_{X_{2}} v_{\varepsilon} d X_{2} d X_{1}+\left|\nabla v_{\varepsilon}\right|_{L^{2}(\Omega)}^{2} \\
& =\int_{\Omega} \nabla u \cdot \nabla v_{\varepsilon} d x \leqslant \frac{1}{2}|\nabla u|_{L^{2}(\Omega)}^{2}+\frac{1}{2}\left|\nabla v_{\varepsilon}\right|_{L^{2}(\Omega)}^{2} .
\end{aligned}
$$

Since $v_{\varepsilon} \in H_{0}^{1}(\Omega)$ and $u \in V \cap W$ in (3.14), for a.e. $X_{1} \in \Pi_{1}$ and a.e. $X_{2} \in \Pi_{2}$ (cf. [4])

$$
\Delta v_{\varepsilon}\left(X_{1}, \cdot\right) \in H_{0}^{1}\left(\Omega_{X_{2}}\right) \quad \text { and } \quad \Delta v_{\varepsilon}\left(\cdot, X_{2}\right) \in H_{0}^{1}\left(\Omega_{X_{1}}\right) .
$$

Thus, we can write (3.15) as

$$
2 \varepsilon \int_{\Pi_{1}} \int_{\Omega_{X_{2}}} \Delta v_{\varepsilon} \Delta_{X_{1}} v_{\varepsilon} d X_{1} d X_{2}+2 \varepsilon \int_{\Pi_{2}} \int_{\Omega_{X_{1}}} \Delta v_{\varepsilon} \Delta_{X_{2}} v_{\varepsilon} d X_{2} d X_{1}+\left|\nabla v_{\varepsilon}\right|_{L^{2}(\Omega)}^{2} \leqslant|\nabla u|_{L^{2}(\Omega)}^{2} .
$$


Hence

$$
2 \varepsilon\left|\Delta v_{\varepsilon}\right|_{L^{2}(\Omega)}^{2}+\left|\nabla v_{\varepsilon}\right|_{L^{2}(\Omega)}^{2} \leqslant|\nabla u|_{L^{2}(\Omega)}^{2} .
$$

It follows that $v_{\varepsilon}$ is bounded in $H_{0}^{1}(\Omega)$. Then - up to a subsequence - its weak limit is in $H_{0}^{1}(\Omega)$, and due to (3.13) this limit is $u$. Thus, $u \in H_{0}^{1}(\Omega)$, which completes the proof.

As is known, we need a pointwise convergence to pass to the limit in the nonlinear term $g\left(\cdot, u_{\varepsilon}\right)$. But the estimates that one has, i.e.,

$$
\left|\nabla_{X_{2}} u_{\varepsilon}\right|_{L^{2}(\Omega)},\left|u_{\varepsilon}\right|_{L^{p}(\Omega)} \text { are bounded, }
$$

are not sufficient to get the pointwise limit of $\left(u_{\varepsilon}\right)_{\varepsilon}$ since the embedding $W \subset L^{2}(\Omega)$ is not compact. So, in this case, the monotonicity hypothesis is necessary, and as an obvious consequence of Theorems 2.2, 2.3 and Corollary 2.1 we have

Corollary 3.2. As $\varepsilon \rightarrow 0$, we have

$$
u_{\varepsilon} \rightarrow \widetilde{u}, \quad \nabla_{X_{2}} u_{\varepsilon} \rightarrow \nabla_{X_{2}} \widetilde{u}, \quad \sqrt{\varepsilon} \nabla_{X_{1}} u_{\varepsilon} \rightarrow 0 \quad \text { in } L^{2}(\Omega)
$$

where $\widetilde{u}$ and $u_{\varepsilon}$ are the solutions to (3.11) and (3.5) respectively. Moreover, if $g$ is strongly monotone, then

$$
u_{\varepsilon} \rightarrow \widetilde{u} \quad \text { in } L^{p}(\Omega)
$$

Remark 3.2. Even if $B$ is not strongly monotone, the first two convergences hold strongly. This is due to the following monotone type inequality:

$$
\left\langle\Delta_{X_{2}} v-\Delta_{X_{2}} u, v-u\right\rangle_{W}+\int_{\Omega}(g(x, v)-g(x, u))(v-u) d x \geqslant\left|\nabla_{X_{2}}(v-u)\right|_{L^{2}(\Omega)}^{2} \quad \forall u, v \in W .
$$

\section{$3.3 \quad p$-Laplacian type problem}

The second application of the abstract theory, in the anisotropic case, is the following quasilinear elliptic equation:

$$
\left\{\begin{array}{l}
-\varepsilon \Delta_{p_{1}, X_{1}} u_{\varepsilon}-\Delta_{p_{2}, X_{2}} u_{\varepsilon}=f \text { in } \Omega \\
u_{\varepsilon}=0 \text { on } \partial \Omega
\end{array}\right.
$$

where $p_{1}, p_{2}>1$ are real constants and $\Delta_{p_{1}, X_{1}}, \Delta_{p_{2}, X_{2}}$ are the $p_{i}$-Laplace operators in $X_{1}$ and $X_{2}$ respectively, i.e.,

$$
\begin{aligned}
& \Delta_{p_{1}, X_{1}} \cdot=\nabla_{X_{1}} \cdot\left(\left.\left|\nabla_{X_{1}} \cdot\right|\right|^{p_{1}-2} \nabla_{X_{1}} \cdot\right) \\
& \Delta_{p_{2}, X_{2}} \cdot=\nabla_{X_{2}} \cdot\left(\left.\left|\nabla_{X_{2}} \cdot\right|\right|^{p_{2}-2} \nabla_{X_{2}} \cdot\right)
\end{aligned}
$$

We assume that $f \in L^{p_{2}^{\prime}}(\Omega)\left(p_{2}^{\prime}\right.$ is the conjugate of $\left.p_{2}\right)$. In this case, we set

$$
V=\left\{\begin{array}{l|l}
u \in L^{p_{1}}(\Omega) & \begin{array}{l}
\nabla_{X_{1}} u \in\left[L^{p_{1}}(\Omega)\right]^{q}, \\
u\left(\cdot, X_{2}\right) \in W_{0}^{1, p_{1}}\left(\Omega_{X_{2}}\right), \text { a.e. } X_{2} \in \Pi_{2}
\end{array}
\end{array}\right\},
$$


equipped with the norm

$$
|v|_{V}=\left|\nabla_{X_{1}} v\right|_{L^{p_{1}}(\Omega)}
$$

and

$$
W=\left\{\begin{array}{l|l}
u \in L^{p_{2}}(\Omega) & \begin{array}{l}
\nabla_{X_{2}} u \in\left[L^{p_{2}}(\Omega)\right]^{n-q}, \\
u\left(X_{1}, \cdot\right) \in W_{0}^{1, p_{2}}\left(\Omega_{X_{1}}\right) \text { a.e. } X_{1} \in \Pi_{1}
\end{array}
\end{array}\right\},
$$

equipped with the norm

$$
|v|_{W}=\left|\nabla_{X_{2}} v\right|_{L^{p_{2}(\Omega)}}
$$

We can easily show that $V$ and $W$ are separable reflexive Banach spaces. Then we define the operators $A: V \rightarrow V^{\prime}$ and $B: W \rightarrow W^{\prime}$ as

$$
A=-\Delta_{p_{1}, X_{1}} \quad \text { and } \quad B=-\Delta_{p_{2}, X_{2}} .
$$

It is easy to see that $A$ and $B$ are coercive, bounded, and hemicontinuous. The monotonicity of $A$ and $B$ is shown by the following lemma (cf. $[2,13])$.

Lemma 3.1. For all $p>1$ and $\xi, \eta \in \mathbb{R}^{n}$

$$
\left(|\xi|^{p-2} \xi-|\eta|^{p-2} \eta\right) \cdot(\xi-\eta) \geqslant c_{p}\{|\xi|+|\eta|\}^{p-2}|\xi-\eta|^{2}
$$

with a constant $c_{p}>0$. If $p \geqslant 2$, then

$$
\left(|\xi|^{p-2} \xi-|\eta|^{p-2} \eta\right) \cdot(\xi-\eta) \geqslant c_{p}|\xi-\eta|^{p}
$$

where $|\cdot|$ is the usual Euclidean norm in $\mathbb{R}^{n}$ and "." is the scalar product.

Thus, the operator $A$ (respectively, $B$ ) is strictly monotone for all $p_{1}>1$ (respectively, $p_{2}>1$ ) and strongly monotone if $p_{1} \geqslant 2$ (respectively, $p_{2} \geqslant 2$ ). The limit problem is defined for a.e. $X_{1} \in \Pi_{1}$ as

$$
\left\{\begin{array}{l}
-\Delta_{p_{2}, X_{2}} \widetilde{u}\left(X_{1}, \cdot\right)=f\left(X_{1}, \cdot\right) \quad \text { in } \Omega_{X_{1}}, \\
\widetilde{u}\left(X_{1}, \cdot\right)=0 \quad \text { on } \partial \Omega_{X_{1}} .
\end{array}\right.
$$

Finally, as in the previous subsection, we can show that $(V \cap W) \subset W_{0}^{1, \min \left(p_{1}, p_{2}\right)}(\Omega)$. More precisely, we have

$$
V \cap W=\left\{\begin{array}{l|l}
u \in L^{\max \left(p_{1}, p_{2}\right)}(\Omega) \mid \begin{array}{l}
\nabla_{X_{1}} u \in\left[L^{p_{1}}(\Omega)\right]^{q}, \quad \nabla_{X_{2}} u \in\left[L^{p_{2}}(\Omega)\right]^{n-q}, \\
\left.u\right|_{\partial \Omega}=0
\end{array}
\end{array}\right\},
$$

which gives a sense to the boundary conditions. Then, by Theorems 2.2, 2.3 and Corollary 2.1, the following assertion holds.

Corollary 3.3. For all $p_{1}, p_{2}>1$,

$$
\begin{aligned}
& u_{\varepsilon} \rightarrow \widetilde{u} \text { in } W, \\
& \varepsilon \nabla_{X_{1}} u_{\varepsilon} \rightarrow 0 \quad \text { in } L^{p_{1}}(\Omega), \\
& \varepsilon \Delta_{p_{1}, X_{1}} u_{\varepsilon} \rightarrow 0 \quad \text { in } V^{\prime}, \\
& \Delta_{p_{2}, X_{2}} u_{\varepsilon} \rightarrow f \text { in } W^{\prime},
\end{aligned}
$$

where $u_{\varepsilon}$ and $\widetilde{u}$ are the solutions to (3.17) and (3.18) respectively. Moreover, if $p_{1} \geqslant 2$, then

$$
\varepsilon^{1 / p_{1}} \nabla_{X_{1}} u_{\varepsilon} \rightarrow 0 \quad \text { in } L^{p_{1}}(\Omega)
$$

and, if $p_{2} \geqslant 2$, then

$$
u_{\varepsilon} \rightarrow \widetilde{u}, \nabla_{X_{2}} u_{\varepsilon} \rightarrow \nabla_{X_{2}} \widetilde{u} \quad \text { in } L^{p_{2}}(\Omega)
$$




\section{Acknowledgments}

The first two authors have been supported by the Swiss National Science Foundation under the contracts \#20-117614/1, \#200021-129807/1, and the last two authors have been partially supported by the National Agency of University Research Development (ANDRU, Algeria). The authors are very grateful to these institutions and to the ICTP (Trieste, Italy) where the paper was completed.

\section{References}

1. B. Brighi and S. Guesmia, "Asymptotic behaviour of solutions of hyperbolic problems on a cylindrical domain," Discrete Contin. Dyn. Syst. Suppl., 160-169 (2007).

2. M. Chipot, Elliptic Equations: An Introductory Course, Birkhäuser, Basel (2009).

3. M. Chipot, "On some anisotropic singular perturbation problems," Asymptotic Anal. 55, No. 3-4, 125-144 (2007).

4. M. Chipot and S. Guesmia, "On the asymptotic behaviour of elliptic, anisotropic singular perturbations problems," Commun. Pure Appl. Anal. 8, No. 1, 179-193 (2009).

5. M. Chipot and S. Guesmia, "On a class of integro-differential problems," Commun. Pure Appl. Anal. 9, No. 5, 1249-1262 (2010).

6. M. Chipot and S. Guesmia, "On some anisotropic, nonlocal, parabolic singular perturbations problems," Appl. Anal. [To appear]

7. M. Chipot and S. Guesmia, "Correctors for some asymptotic problems," Proc. Steklov Inst. Math. 270, 263-277 (2010).

8. S. Guesmia, "Asymptotic behaviour of elliptic boundary value problems with small coefficients," Electron. J. Differ. Equations 59, 1-13 (2008).

9. S. Guesmia, Etude du comportement asymptotique de certaines équations aux dérivées partielles dans des domaines cylindriques, Thèse Université de Haute Alsace (2006).

10. S. Guesmia and A. Sengouga, "Anisotropic singular perturbation of hyperbolic problems," Appl. Math. Comput. 217, No. 22, 8983-8996 (2011).

11. E. Sanchez-Palencia, "Asymptotic and spectral properties of a class of singular-stiff problems," J. Math. Pures. Appl. 71, 379-406 (1992).

12. S. Zhang, "Equivalence estimates for a class of singular perturbation problems," $C$. $R$. Acad. Sci. Paris, Ser. I 342, 285-288 (2006).

13. J. L. Lions, Perturbations Singulières dans les Problèmes aux Limites et en Contrôle Optimal, Lect. Notes Math. 323, Springer, Berlin (1973).

14. J. L. Lions and G. Stampacchia, "Variational inequalities," Commun. Pure Appl. Math. 20, 493-519 (1967).

15. G. Stampacchia, Variational inequalities, Proc. Nato, Venezia, pp. 101-192 (1968).

Submitted on May 18, 2011 